\title{
Potentials of mean force in acidic proton transfer reactions in constrained geometries
}

\author{
Jordi Martía* \\ ${ }^{a}$ Department of Physics, Technical University of Catalonia-Barcelona Tech, B5-209 Northern Campus \\ UPC. Jordi Girona, 1-3. 08034 Barcelona. Catalonia. Spain.
}

(September2016)

\begin{abstract}
Free energy barriers associated to the transfer of an excess proton in water and related to the potentials of mean force in proton transfer episodes have been computed in a wide range of thermodynamic states, from low density amorphous ices to high temperature liquids under the critical point for unconstrained and constrained systems. The latter were represented by setups placed inside hydrophobic graphene slabs at the nanometric scale allocating a few water layers, namely one or two in the narrowest case. Waterproton and carbon-proton forces were modelled with a Multi-State Empirical Valence Bond method. As a general trend, a competition between the effects of confinement and temperature is observed on the local hydrogen-bonded structures around the lone proton and, consequently, on the mean force exerted by its environment on the water molecule carrying the proton. Free energy barriers estimated from the computed potentials of mean force tend to rise with the combined effect of increasing temperatures and the packing effect due to a larger extent of hydrophobic confinement. The main reason observed for such enhancement of the free energy barriers was the breaking of the second coordination shell around the lone proton.
\end{abstract}

Keywords: Potential of mean force, acidic proton transfer, graphene slab, Multistate Empirical Valence Bond, Molecular Dynamics.

\section{Introduction}

Proton transfer (PT) in water and aqueous solutions is one of fundamental processes in nature, playing a central role in processes such as water autoionization,[1] molecular reactions in aerosols,[2] or in energy conversion processes like photosynthesis and in cellular respiration, [3] to mention only a few relevant examples. The structure of excess protons in bulk water has been well understood quite recently, [4] basically from computer simulations and from experimental measurements as well. The mechanism which has gained general agreement within the community of chemical physicists indicates that the aqueous proton can be casted out in terms of a "default" propagating within the three-dimensional hydrogen-bond (HB) network of water, carrying out a (first) water solvation shell formed by one single water (hydronium, $\mathrm{H}_{3} \mathrm{O}^{+}$), two water molecules (Zundel dimer, $\mathrm{H}_{5} \mathrm{O}_{2}^{+}$) or three water molecules (Eigen cation, $\mathrm{H}_{9} \mathrm{O}_{4}^{+}$), under continuous interconversion among such three species. The classical model of the dynamics of the proton was introduced by Von Grotthuss long time ago,[5-7] consisting in a mixed mechanism of rotation and jump between neighboring molecules, using the wires formed by hydrogen-bonding. After long time of analysis and debate from both experimental and theoretical studies[8-24] a general consensus arose, based on the fact that the dynamics of the lone proton in water is directly associated with dynamics of the HB network, so that any external influence on the HB structure of water will significantly modify the 
dynamics of the acidic proton.

When turning to the transport of the a lone proton within complex, strongly confined aqueous environments, the characteristics of such species are still far from being well understood. Often protons are conducted through restricted spaces, such as in the case of protons within water channels like those of nafion membranes in hydrogen fuel cells, [25-27] for protons in tiny water nanopools embedded inside proteins and peptides,[28] for protons near alumina surfaces[29] or inside biomembranes. [22] Nevertheless, despite the large scientific and industrial relevance of confined protons, the mechanisms of proton conduction in confined spaces are still a largely unexplored area of research, essentially due to the limited number of experimental techniques accurate enough and also the lack of detailed predictions from the theory and simulation side. As a general fact it has been observed that under restricted geometries local structure and dynamics of the proton suffer drastic changes from those at bulk states[30, 31].

Given the complexity of the phase diagram of water[32, 33], computational studies of aqueous lone protons are usually reported for a few selected thermodynamical states. Multi-State Empirical Valence Bond (MS-EVB) calculations of PT in water inside carbon nanotubes[34] confirmed previous results [35] and revealed that PT rates in severe confinement are about one order of magnitude faster than in bulk. Recently Wolf and Groenhof[36] proposed a new classical model with explicit proton transfer that has provided a very efficient computational tool using classical force fields and capturing key aspects of the phenomenon. More accurate but very expensive simulations made with ab initio Molecular Dynamics (MD) of water inside nanochannels[37, 38] reported different mobilities for hydroxyl $\mathrm{OH}^{-}$and hydronium $\mathrm{H}_{3} \mathrm{O}^{+}$ions, depending essentially on the size of the tubes and the degree of functionalization of the tubes. Mahadevan and Garofalini[39] introduced a dissociative water potential including three-body terms able to reproduce the liquid-vapor coexistence curve. The reliability of such a potential model has been recently examined and a good agreement of the local structure and dynamical properties of the lone proton with ab-initio data has been found[40]. Finally, Car-Parrinello simulations by Bankura and Chandra[41] indicated that in water inside narrow two-dimensional environments the lone proton is normally solvated as the Eigen cation and that PT rates are much lower than in the quasi-one-dimensional case described above. However, few works have focussed on the free energy barriers of PT and their relationship to the local structure of the proton[42]. Although there is plenty of information on microscopic properties of lone protons in constrained water, the influence of a variety of factors on them, from variations in thermal energy to hydrophobicity effects makes the problem very difficult to handle. Based on a previous work of computer simulations of excess protons confined in narrow nanometric channels at room conditions[43], I will report in the present paper the potentials of mean force between the instantaneous protonated water molecule, i.e. the water molecule carrying the proton, and its closest neighbors in an attempt to evaluate the influence of temperature and confinement together on the likeliness of the transfer episodes and on the characteristics of the energy barriers associated with acidic PT.

\section{Methods}

The computational experiments reported in this study have been performed by means of a MS-EVB approach combined with MD simulations. The implementation of such kind of methodology has been widely described in the literature[44-58], so that here I will restrict myself to give a fair but schematic description of the main features of the method. Full details can be found in a previous work[43] and in references therein.

The systems considered in the present work consisted in: (1) a quantum particle (excess proton) and (2) a classical bath (water) formed by 125 water molecules. The EVB method assumes that the Born-Oppenheimer potential energy surface $\epsilon_{0}(\{\mathbf{R}\})$ driving the dynamics of the nuclei with coordinates $\{\mathbf{R}\}$ can be obtained from the lowest instantaneous eigenvalue of a EVB Hamiltonian 
$\hat{\mathrm{H}}_{\mathrm{EVB}}(\{\mathbf{R}\})$. This Hamiltonian is represented in terms of a basis set $\left\{\left|\phi_{i}\right\rangle\right\}$ of diabatic (localized) states. For a lone proton in water, these diabatic states are associated to configurations with the $\mathrm{H}^{+}$located in a given water oxygen. The ground-state $\left|\psi_{0}\right\rangle$ of the Hamiltonian satisfies:

$$
\hat{\mathrm{H}}_{\mathrm{EVB}}\left|\psi_{0}\right\rangle=\epsilon_{0}(\{\mathbf{R}\})\left|\psi_{0}\right\rangle
$$

It can be written as a linear combination of diabatic states $\left|\phi_{i}\right\rangle$ as:

$$
\left|\psi_{0}\right\rangle=\sum_{i=1}^{N_{b}} c_{i}\left|\phi_{i}\right\rangle,
$$

with $N_{b}$ being the number of basis sets and the potential energy surface is given by:

$$
\epsilon_{0}\{\mathbf{R}\}=c_{i} c_{j} h^{i j}(\{\mathbf{R}\})
$$

with $h^{i j}$ standing for the matrix elements of the EVB Hamiltonian. In the framework of EVB methods, off-diagonal elements $h_{i j}$ can be casted out in terms of nuclear coordinates, achieving an excellent agreement with results from full quantum calculations. The parameterization employed in this work follows was proposed by Schmitt, Voth et al. and it has been proven to be very successful in a wide variety of cases[51-55]. The newest version of this procedure (MS-EVB 3.2) was recently published[58]. Within this framework, Schmitt et al. were able to reproduce geometries and energetics of the most important protonated water clusters (such as $\mathrm{H}_{5} \mathrm{O}_{2}^{+}, \mathrm{H}_{7} \mathrm{O}_{3}^{+}$and $\mathrm{H}_{9} \mathrm{O}_{4}^{+}$), obtained from ab initio calculations. Diagonal elements $h_{i i}$ include contributions from stretching and bending intramolecular interactions within the tagged $\mathrm{H}_{3} \mathrm{O}^{+}$and also inside the rest of water molecules, which are modeled using a flexible TIP3P force field[59]. Diagonal elements also include intermolecular interactions such as those between hydronium-solvent and solvent-solvent. Conversely, off-diagonal elements $h_{i j}$ account for the coupling between diabatic states $i$ and $j$ and have been modeled including interatomic contributions within a particular $\mathrm{H}_{5} \mathrm{O}_{2}^{+}$Zundel dimer spanned by states $\left|\phi_{i}\right\rangle$ and $\left|\phi_{j}\right\rangle$ plus Coulomb interactions between the dimer and the rest of solvent. A complete list of parameters is provided in Ref.[53]. Oxygen-carbon and hydrogen-carbon forces were modeled by means of Lennard-Jones forces with the same parameterization employed previously. [60] From Lorentz-Berthelot mixing rules, the size and energy crossed parameters were $\sigma_{O C}=3.28 \AA \sigma_{H C}=2.81 \AA \varepsilon_{O C}=0.389 \mathrm{~kJ} / \mathrm{mol} \varepsilon_{H C}=0.129 \mathrm{~kJ} / \mathrm{mol}$.

The construction of the EVB Hamiltonian is usually performed by a series of steps. First, the water closest to the excess proton is identified. This particular water forms the initial pivot $\mathrm{H}_{3} \mathrm{O}^{+}$ complex and the first diabatic state. The rest of the diabatic states are chosen in a tree-like construction via a hydrogen-bond connectivity pattern. The criterion to establish a hydrogen bond is as follows: The maximum oxygen acceptor-proton donor distance is of $2.8 \AA$ and a minimum threshold value of the $\mathrm{H}-\mathrm{O}-\mathrm{O}$ angle of $30^{\circ}$ is taken. All molecules lying in up to the third solvation shell and showing a connecting path with the original pivot are included in the construction of the $L \times L$ EVB Hamiltonian matrix and finally this matrix is properly diagonalized. In general, about $L \sim 10-20$ diabatic states for the connectivity pattern have been found. In all cases, fluctuations in the total energy never surpassed $1 \%$. At each step, proton transfer was made possible by reassigning the pivot oxygen label to the instantaneous state exhibiting the largest $c_{i}^{2}$ coefficient; from this state, the list of participating VB states was reconstructed using the connectivity branching procedure mentioned above. Once the EVB matrix was formed, ground-state eigenvectors and Hellmann-Feynman forces $\mathbf{F}_{k}$ on all particles $(k=1, \ldots, N)$ were computed by means of: 


$$
\mathbf{F}_{k}=-\left\langle\psi_{0}\left|\frac{\partial \hat{H}_{E V B}}{\partial \mathbf{x}_{k}}\right| \psi_{0}\right\rangle=-c_{i} c_{j} \frac{\partial \hat{H}_{E V B}^{i j}(\mathbf{x})}{\partial \mathbf{x}_{k}}
$$

where $\mathbf{x}_{k}$ stand for any spatial coordinate. Finally, dynamics of the nuclei of mass $M_{k}$ is given by the classical (Newtonian) equation of motion:

$$
M_{k} \frac{\mathrm{d}^{2} \mathbf{R}_{k}}{\mathrm{~d} t^{2}}=-c_{i} c_{j} \nabla_{\mathbf{R}_{k}} h^{i j}(\{\mathbf{R}\}) .
$$

The systems were fully unconstrained or contained inside a flat graphene slab with variable interplate distances $d=3.1,1.5,0.7 \mathrm{~nm}$. All simulations corresponded to microcanonical runs at the temperatures of $T=100,200,300,400,500$ and $600 \pm 20 \mathrm{~K}$. In all cases, fluctuations in energy were only $\sim 1 \%$ of the averaged value and no energy drift was observed. To give a few precise values, I observed that for the interplate distance of $1.5 \mathrm{~nm}$ the average in total energy was of $\langle E\rangle=175.2 \pm 1.4 \mathrm{kcal} / \mathrm{mol}$ whereas the average in temperature was of $\langle T\rangle=298.3 \pm 1.6 \mathrm{~K}$. The density of the system was increased from $0.02 \AA^{-3}$ (3.1 nm wide slab) to $0.07 \AA^{-3}$ (0.7 nm wide slab). This allowed to record some proton transfers in all cases. The boxlength along $\mathrm{X}$ and $\mathrm{Y}$ coordinates was of $15.6 \stackrel{\circ}{A}$ in all cases, whereas the simulation boxlength along the Z-coordinate was enlarged five times the value of the $\mathrm{X}(\mathrm{Y})$ direction, in order to avoid interactions between image graphene sheets[61]. Finally, our time step was set to $\Delta t=0.5$ fs for all simulations. I considered equilibration periods of approximately $20 \mathrm{ps}$, followed by trajectories of more than $0.25 \mathrm{~ns}$, used to obtain meaningful statistical properties. Long ranged forces (Coulomb interactions) were handled by Ewald sum techniques[62], using a uniform neutralizing background charge in all cases. This is satisfactory enough for a system including a small charge as the lone proton, whereas in extended systems such as proteins of membranes in water, it has been reported that the use of a counterion would be in order[63].

\section{Structure of the lone hydrated proton}

A solvated ion in water is known to produce a huge anomaly in the local tetrahedral structure of the liquid. Similarly, the excess proton also creates a disruption in the local hydrogen-bond structure, although in a smaller scale. However, this fact together with the existence of the Grotthuss mechanism driving the dynamics of the proton leads to a direct connection between proton-water forces, the local solvation structure and the proton transfer mechanism. So, after the transfer of the proton between two neighboring water molecules a subtle balance between three relevant structures must be sustained: the proton attached to a single water (single hydronium, $\mathrm{H}_{3} \mathrm{O}^{+}$), the so-called Zundel dimer $\left(\mathrm{H}_{5} \mathrm{O}_{2}\right)^{+}[64]$ and the three-coordinated hydronium $\left(\mathrm{H}_{9} \mathrm{O}_{4}\right)^{+}$known as the Eigen complex[65]. It is widely accepted that $\mathrm{PT}$ is the result of the continuous interconversion of the three above mentioned structures, with percentages of each depending on the thermodynamic conditions of the system. In most cases, continuous interconversions between the Zundel and Eigen complexes generate a hybrid $\left(\mathrm{H}_{9} \mathrm{O}_{4}\right)^{+} /\left(\mathrm{H}_{5} \mathrm{O}_{2}\right)^{+}$structure[66, 67]. As a first approach to structural analysis, snapshots of the local water structure at two relevant cases are shown in Fig. 1. For the sake of clarity, I have only included those molecules having the largest weighting coefficients $c_{i}$ from Eq. 2 (of the order of 10 molecules), which usually are the water molecules belonging to the first and second solvation shells of the instantaneous hydronium species. From this picture a first direct indication is seen that the local environment of the proton is essentially formed by a few water molecules, essentially the Eigen cation (image at left), and that under strict confinement 
(interplate distance of $0.7 \mathrm{~nm}$, image at right) the solvating waters no longer surround the proton but they allow it to stay close enough to the interfacial graphene layers.

Every transfer of the lone proton between two water molecules will involve changes in pivot oxygen-water oxygen $\left(\mathrm{O}^{*}-\mathrm{O}\right)$ and pivot oxygen-water hydrogen $\left(\mathrm{O}^{*}-\mathrm{H}\right)$ distances and changes of the local hydrogen connectivity pattern between the complex at its closest solvation shells. Both temperature and confinement are expected to have observable effects on the local proton structure. As usual solvation structures can be analyzed by means of local pivot oxygen-water radial distribution functions $(\mathrm{RDF})$ given by:

$$
g_{\mathrm{O}^{*} \mathrm{O}}(r)=\frac{V}{4 N \pi r^{2} \Delta r}\left\langle n_{\mathrm{O}^{*} \mathrm{O}}(r)\right\rangle,
$$

where $n_{\mathrm{O}^{*} \mathrm{O}}(r)$ is the number of solvent water molecules located in a spherical shell of width $\Delta r$ and radius $r$ centered at the pivot water, $N$ is the total number of water molecules in the system and $V$ is the total volume. The brackets indicate both ensemble and time average. The RDF of pivot-water oxygens are represented in Fig. 2. I selected three representative temperatures: 200, 300 and $600 \mathrm{~K}$ for all interplate distances compared to the unconstrained system. However, since the number of proton transitions is rather low for some of the selected thermodynamical states, the collected statistics during simulation runs are not optimal, producing fluctuating RDF. The selected temperatures represent states of: local density amorphous ice (LDA, $200 \mathrm{~K}$ ), ambient conditions $(300 \mathrm{~K})$ and high-temperature sub-critical conditions $(600 \mathrm{~K})$, according the approximate phase diagram for the force field employed in the present work (see Ref. [24]). All figures show a structure formed by a marked first peak and a secondary maximum. The first maximum, centered around $2.5 \AA$ in all cases, should be attributed to the first solvation shell of the proton. The location of this maximum is in good agreement with the findings of Bankura and Chandra[41] and also with the RDF reported in the work of Lockwood and Garofalini[42] where the authors show that, within the framework of their potential model and at ambient conditions, the first maximum in $\left(\mathrm{O}^{*}-\mathrm{O}\right)$ is located at slightly smaller distances that the maximum of oxygen-oxygen RDF when all oxygens are considered. This is called "inward shift" and indicates that the first solvation shell of the proton is significantly smaller than that of oxygens in pure water. This fact has been recently related to three-body interactions by Wiedemair et al.[40]. Further, this feature was also previously observed in our simulations[43] and it suggests the equivalence of the present MS-EVB simulations with the MD calculations with the dissipative potential[42] regarding the meaning of local proton structures. In our case, this first maximum of the RDF is essentially invariable, regardless of $T$ or $d$. This fact gives an indication of the strength of the proton to create a first solvation shell which remains virtually invariable at all conditions. The only remarkable fact about it is that at the low and moderate temperatures $(200$ and $300 \mathrm{~K})$ the size of the first water shell coordinating the proton in the confined systems tends to become smaller than in the unconstrained case, whereas at $600 \mathrm{~K}$ any difference cannot be seen.

Looking at the secondary maximum, both the interplate separation and the temperature play a subtle role in its location. First of all, this feature is located at distances closer than those reported elsewhere[41, 42] including the observation (see [43]) of the compression of the local coordination of the proton (the "inward shift" by Lockwood and Garofalini[42]). Second, at the LDA state the maximum is centered at $4.2 \AA$ in all cases but moves to $3.5 \AA$ when $d=0.7 \mathrm{~nm}$. In the particular case of $d=0.7 \mathrm{~nm}$, the distance between the two plates is so short that, in average, there is only room available for two water layers. This suggest that compressing the amorphous ice would break some hydrogen bonds and let the coordination shell of the proton become smaller. At room temperature, the maximum shifts backwards from $4.75 \AA$ (unconstrained case) to $\sim 4 \AA$ (confined cases), so that the effect of confinement is clear: it forces the local environment of the proton to become more packed. Finally, at the high temperature case, the second maximum is clearly 
milder than at lower temperatures, showing a moderate shift from $4.5 \AA$ (unconstrained case) to $\sim 4.2 \AA$ (confined cases). There it can distinguished how this maximum is enhanced at the smallest interplate distance, suggesting a larger extent of localization of second shell of the proton. From all these findings, a general tendency can be drawn: the local cluster around hydronium tends to become smaller as confinement becomes more important. This larger extent of water localization is similar to the case of cubic ice[10, 11].

\section{Potentials of mean force for proton transfer}

Once the local structure around the lone proton has been obtained, a common way to analyze the microscopic forces acting on it is by means of the so-called potential of mean force $P M F(r)$, that can be readily obtained from the $\mathrm{RDF}$ :

$$
P M F(r)=-k_{B} T \ln g_{\mathrm{O}^{*} \mathrm{O}}(r),
$$

where $k_{B}$ is Boltzmann constant. Since in our case the RDF reported in Fig. 2 show non-negligible fluctuations, I employed a procedure based on neural network and data mining procedures[68] in order to improve the statistics in the RDFs and get meaningful PMF. This leads to an accurate estimation of the errors associated to the PMFs, that were included in Fig. 3 where, as a benchmark, the reference case of ambient conditions is reported for three selected states: unconstrained and constrained inside graphene channels of width $3.1,1.5$ and $0.7 \mathrm{~nm}$. The comparison of these results to those of Lockwood and Garofalini indicates that the size of the free energy barriers is in overall good agreement: in the present work the obtained value is of about $\sim 4 k_{B} T=2.3 \mathrm{kcal} / \mathrm{mol}$ (at $300 \mathrm{~K}$ ) for the unconstrained setup, that is of the same order of magnitude of the values reported in Ref.[42] (between 0.8 and $1.2 \mathrm{kcal} / \mathrm{mol}$ ).

Here it is important to mention that the reaction coordinate considered in the present work is directly the $\mathrm{O}^{*}-\mathrm{O}$ distance, whereas in the work of Lockwood and Garofalini[42] the $\mathrm{O}_{-}-\mathrm{O}$ distance is used as a selector of first neigbour waters, with the difference of distances between the lone proton and $\mathrm{O}^{*}$ or $\mathrm{O}$ taken as the reaction coordinate. We should mention that in our case the computed PMFs using exactly the same coordinate as in Ref.[42] lead to energy barriers practically equal as those reported here, within $10 \%$ of the absolute values of the heights of energy barriers. At this point, I would like to mention that it was made clear long time ago that the use of one-dimensional (and usually geometrical) reaction coordinates is simply an approximation to the real ones[1], which may be in general multidimensional, presumably involving a limited number of water molecules and, eventually coordinates or distances to the confining walls. So, since the determination of the true reaction coordinate for constrained aqueous protons is out of the scope of this paper, I will consider the $\mathrm{O}^{*}-\mathrm{O}$ distance as an order parameter useful to account for mean forces between instantaneous hydronium and solvating waters. So, the free energies reported should be directly associated to the exchange of identities between the instantaneous hydronium and one of the (usually three) solvating waters. This will not in general correspond to the full mean force for proton transfer but to part of it, since we should bear in mind that before a full PT episode occurs and the identities of the two waters are changed, there exists a time interval where the proton rattles in between the two given waters, usually called "resonance". This "transient" time (that can reach values of the order of $1 \mathrm{ps}$ ), is a fundamental part of the full PT episode (see for instance Ref. [17]). Further, since the exchange between proton's first and second coordination shells of "external" water molecules, i.e. not directly related to PT, is allowed our calculations are reporting energy barriers affected by such water-water exchange. This fact could account for the deviation between the findings of the present work and those of Ref.[42].

A deep minimum indicating one stable state for the aqueous proton is observed in all cases and 
located around $2.5 \AA$, as expected. This corresponds to the well localized proton at its ground-state configuration, being a Zundel dimer or an Eigen complex in most cases. Further, it can be due to a bare hydronium species for the lowest coordinated states, namely those at high temperatures or low densities[24]. Then a free-energy barrier of height $E_{H} 2.5 k_{B} \mathrm{~T}$ is observed for the unconstrained system, located around $2.9 \AA$. Such a barrier shifts backwards and is centered around $2.7 \AA$ for the constrained systems with its height rising up to $\sim 5.7 k_{B} \mathrm{~T}$ for the state at the narrowest interplate distance of $d=0.7 \mathrm{~nm}$. This clearly indicates the increase in the energetic cost of water-hydronium exchange at strongly constrained systems, rising about a factor 2 compared to the unconfined case. A second minimum close to $4.5 \AA$ is also seen (varying between $4.6 \AA$ for the unconstrained system and $3.9 \AA$ when the aqueous proton is constrained inside the $d=0.7 \mathrm{~nm}$ channel). This minimum should be assigned to a much milder associated state, related with the second solvation shell of the proton. This state can be attributed to the super-structure formed by the main protonated complex (when Zundel and Eigen structures exist) and its water shell, given the low buy non-vanishing weights $c_{i}$ (see Eq.2) of the diabatic states in all cases (between 10 and 20). This interpretation would be consistent with the fact that at $300 \mathrm{~K}$, all states but the one with $d=0.7 \mathrm{~nm}$ have room enough to allocate two and, eventually, more solvation shells of the central protonated complex. In the latter case the lack of space constrains the cluster around the proton, making it smaller and containing less diabatic states with $c_{i} \neq 0$.

Under temperature changes, the PMFs are reported in Fig. 4, namely the corresponding to the LDA state at $200 \mathrm{~K}$ (a) and to the highest temperature of $600 \mathrm{~K}$ (b). In the case (a), the main features are quite similar to those reported above, but some significant changes are seen. So, the first maximum has a similar height in all cases but for the case with $d=0.7 \mathrm{~nm}$, with heights of around $4 k_{B} \mathrm{~T}$ and located around $r=2.8 \AA$. This means that at LDA ices and when the system is unconstrained, the barrier to surmount is higher than at room temperature conditions. However, the effect of confinement is rather unimportant when $d=3.1$ and $1.5 \mathrm{~nm}$, whereas in the extremely constrained setup at $d=0.7 \mathrm{~nm}$, a very narrow shape is observed, together with a short barrier of $\sim 2 k_{B} \mathrm{~T}$. This is an indication of an easier, cheaper energetic cost for water-hydronium exchange due to the quasi-two-dimensional geometry of the local cluster around the excess proton. The second minimum of the PMF is also observed at $200 \mathrm{~K}$, in a similar fashion to the case of ambient conditions.

In the case (b) of high temperatures, the biggest differences with the findings reported above at $300 \mathrm{~K}$ have to see with the height of the main energy barrier. At the unconstrained and mildly constrained setups very short barriers under $1 k_{B} \mathrm{~T}$ are observed, located around $r=2.7 \AA$. Again the case of $d=0.7 \mathrm{~nm}$ involves a large energy barrier of $\sim 4.6 k_{B} \mathrm{~T}$, suggesting that $\mathrm{PT}$ episodes started from water-hydronium exchanges will be happen much less commonly. The calculation of the PT rates (see Ref. [43]) delivered rates of $1.2 \mathrm{ps}^{-1}$ for the unconstrained system, whereas this rate decreased to $\sim 0.01 \mathrm{ps}^{-1}$ when $d=0.7 \mathrm{~nm}$. Now the reason for such drop is clear that has to be charged to the large size of the energy barrier. Concerning the second maximum, I should simply remark that its existence (located around $r=4.3-4.4 \stackrel{\circ}{A}$ ) is rather doubtful, due to the large fluctuations in the PMF.

In summary, a general trend has arisen: constraining the system requires larger free energy for the proton to be exchanged between neighboring waters. Experimental data revealed values of the order of $-10 \mathrm{~kJ} / \mathrm{mol}$ for the activation energy of PT at the surface of polycrystalline ice films (at $135 \mathrm{~K}$ ), when the PT is mediated by hydroxyl ions (reported by Moon et al. [69, 70] and Kim et al.[71] from reactive ion scattering) and for PT in pure water (Luz and Meiboom[72], obtained by proton magnetic relaxation measurements). The values of the energy barriers reported in the present work (around $2.3 \mathrm{kcal} / \mathrm{mol} \sim 10 \mathrm{~kJ} / \mathrm{mol}$ for the unconstrained system at $300 \mathrm{~K}$, see above) agree well with the order of magnitude of the experimental measurements and with those obtained by Lockwood and Garofalini[42] and from previous estimation of the PT activation energy[43] although they cannot be properly considered as equivalent to activation energies of PT. 


\section{Conclusions}

In this study a mixed Molecular Dynamics and Multi-State Empirical Valence Bond study of the structure and free energies of an acidic proton in bulk water and in water inside a graphene slab of variable width has been reported. The range of temperatures included states from LDA ices to liquid water up to sub-critical high-temperature states. A suitable Hamiltonian for the semiclassical system formed by a quantum-like particle (the lone proton) embedded in a sea of purely classical flexible TIP3P waters was constructed. The main findings have revealed the enhancement of the local structure of the proton in LDA ices and its progressive reduction as temperature rises. In agreement with other works, it has been observed a compression of the local coordination of the proton ("inward shift" [42]).

The potentials of mean force were obtained from radial distribution functions and should be attributed to the free energy required by one water solvating hydronium to be exchanged with the hydronium and occupy its place as the proton host. These PMF revealed that free energy barriers tend to rise with the combined effect of increasing temperatures and the packing effect due to the hydrophobic confinement. Both conditions favor the breaking of the second coordination shell of the proton and reduce the likeliness of PT. The comparison of the energy barriers for waterhydronium exchange (of the order of $10 \mathrm{~kJ} / \mathrm{mol}$ ) indicates a general good agreement with available experimental data of activation energies for PT[69-72] as well as with results previously reported by other authors on equivalent PMFs barriers for proton transfer in water using a different approach, based on the so-called dissociative water potential[42].

\section{Acknowledgement}

The author gratefully acknowledges financial support from Spanish MINECO for grant FIS201239443-C02-01. 

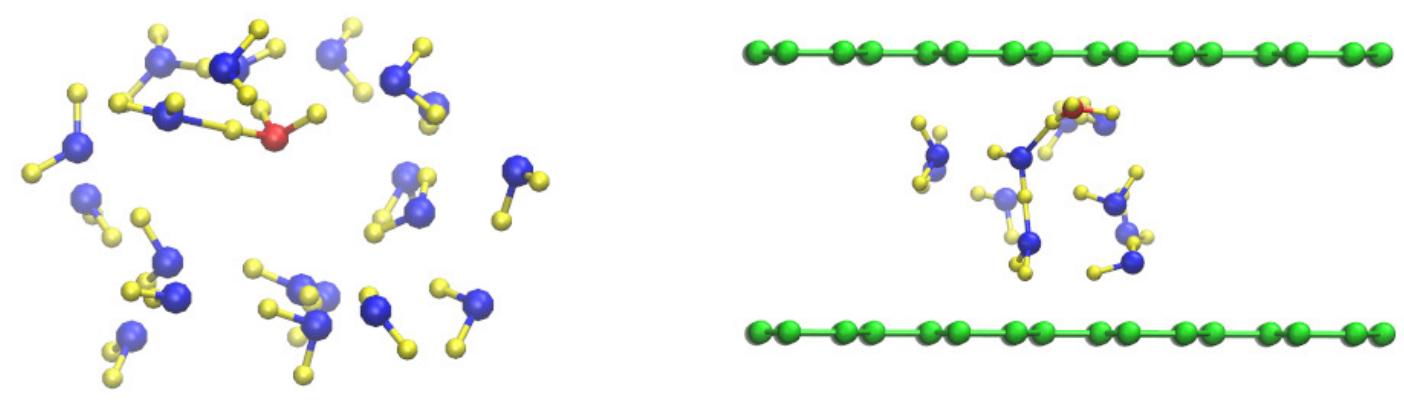

Figure 1. Snapshots of local structures around the lone proton at $300 \mathrm{~K}$. Only the diabatic states with largest $c_{i}$ coefficients (see Eq.2) are shown. Unconstrained system (left) and system between two graphene plates, separated $0.7 \mathrm{~nm}$ (right). Oxygen (blue), hydrogen (yellow), pivot oxygen (see text, red) and carbon (green).

\section{Figures}




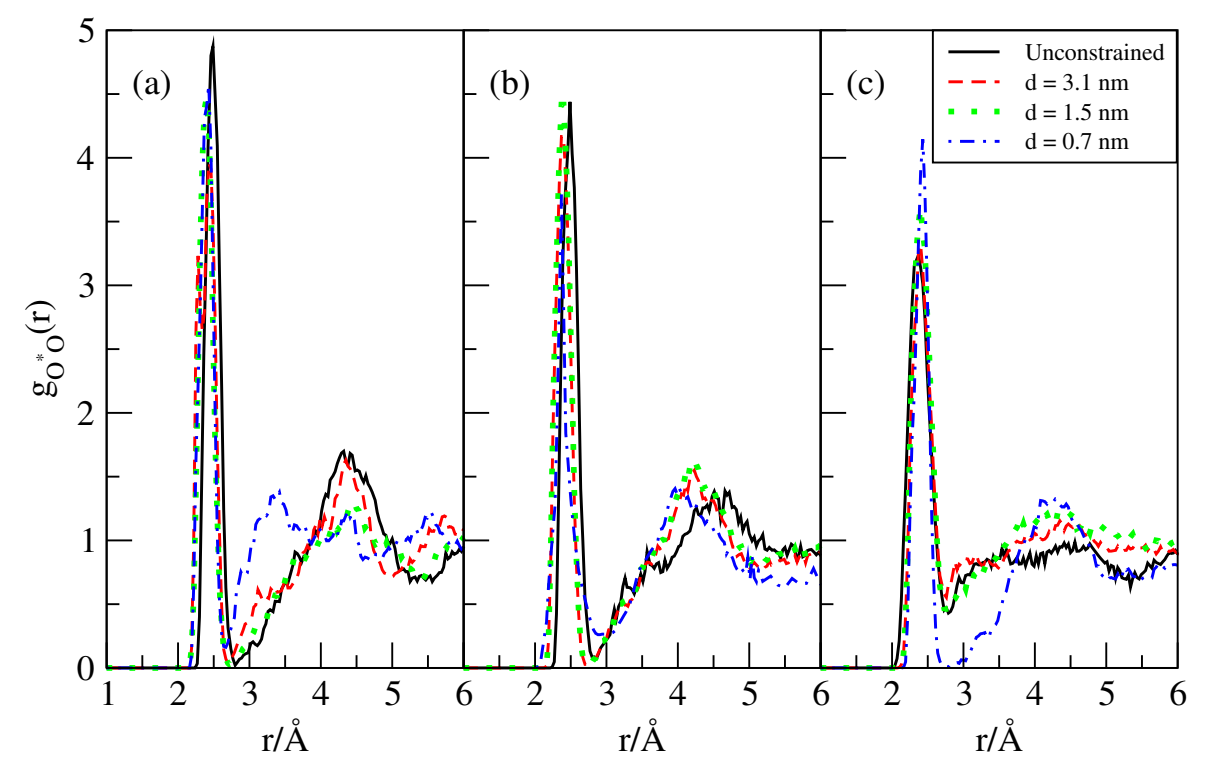

Figure 2. Pivot oxygen-solvent oxygen radial distribution functions at three temperatures: $200 \mathrm{~K}$ (a), $300 \mathrm{~K}$ (b) and 600 $\mathrm{K}(\mathrm{c})$.

\section{References}

[1] Geissler PL, Dellago C, Chandler D, Hutter J, Parrinello M. Autoionization in liquid water. Science. 2001;291:2121-2124.

[2] Bianco R, Hynes JT. A theoretical study of the $\mathrm{H} 2 \mathrm{SO} 4+\mathrm{H} 2 \mathrm{O} \mathrm{HSO} 4+\mathrm{H} 3 \mathrm{O}+$ reaction at the surface of aqueous aerosols. Theor. Chem. Acc. 2004;111:182-187.

[3] Müller A, Ratajczak H, Junge W, Diemann E. Electron and proton transfer in chemistry and biology: Elsevier Science Publishers; 1992.

[4] Marx D, Tuckerman ME, Hutter J, Parrinello M. The nature of the hydrated excess proton in water. Nature. 1999;397:601-604.

[5] von Grotthus CJT. Sur la décomposition de l'eau et des corps qu'elle tient en dissolution à l'aide de l'électricité galvanique. Ann. Chim. (Paris). 1806;58:54-74.

[6] Agmon N. The Grotthuss mechanism. Chem. Phys. Lett. 1995;244: 456-462.

[7] Cukierman S. Et tu, Grotthuss! and other unfinished stories. Biochim. et Biophys. Acta-Bioenergetics. $2006 ; 1757: 876-885$.

[8] Tuckerman ME, Marx D, Klein ML, Parrinello M. On the quantum nature of the shared proton in hydrogen bonds. Science. 1997;275:817-820.

[9] Day TJ, Schmitt UW, Voth GA. The mechanism of hydrated proton transport in water. J. Am. Chem. Soc. 2000;122:12027-12028.

[10] Kobayashi C, Saito S, Ohmine I. Mechanism of fast proton transfer in ice: Potential energy surface and reaction coordinate analyses. J. Chem. Phys. 2000;113:9090-9100.

[11] Kobayashi C, Saito S, Ohmine I. Mechanism of proton transfer in ice. II. Hydration, modes, and transport. J. Chem. Phys. 2001; 115:4742-4749.

[12] Walbran S, Kornyshev AA. Proton transport in polarizable water. J. Chem. Phys. 2001;114:1003910048. 


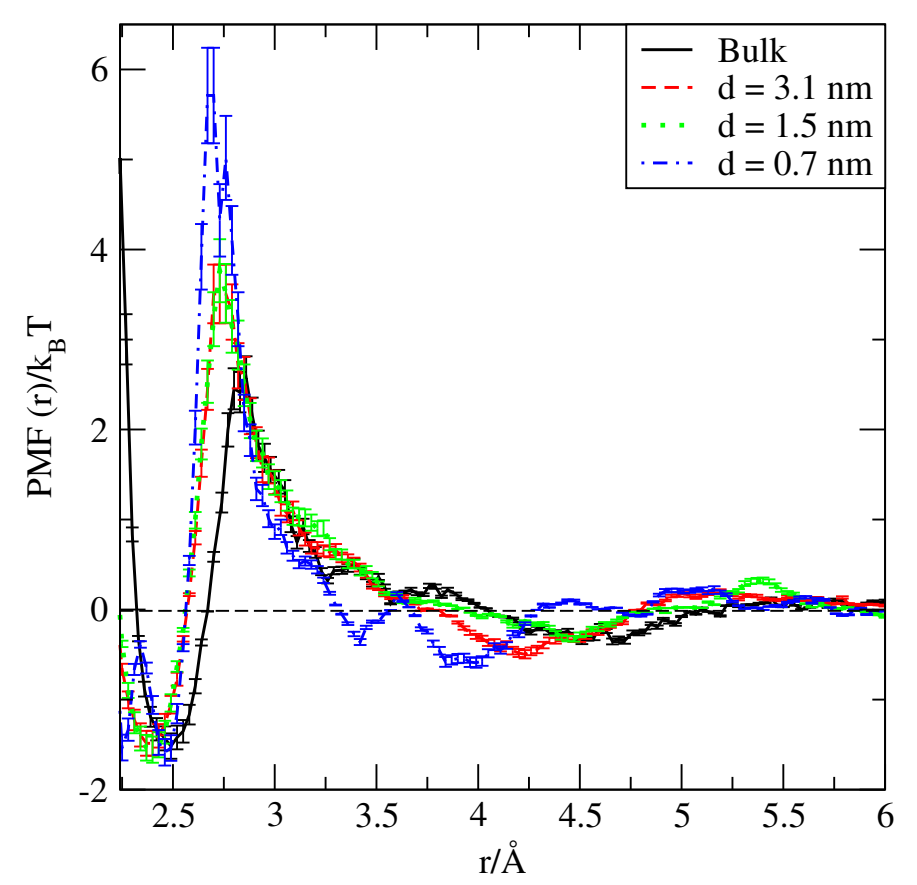

Figure 3. Potentials of mean force proton-water (in $k_{B} T$ ) at $300 \mathrm{~K}$ for unconstrained water and for water inside a graphene slab of variable width. The estimated errors were obtained from neural network and data mining techniques (see text).

[13] Tuckerman ME, Marx D, Parrinello M. The nature and transport mechanism of hydrated hydroxide ions in aqueous solution. Nature. 2002;417:925-929.

[14] Bakker HJ, Nienhuys HK. Delocalization of protons in liquid water. Science. 2002;297:587-590.

[15] Kornyshev AA, Kuznetsov AM, Spohr E, Ulstrup J. Kinetics of Proton Transport in Water. J. Phys. Chem. B. 2003;107:3351-3366.

[16] Botti A, Bruni F, Imberti S, Ricci MA, Soper AK. Ions in water: The microscopic structure of a concentrated HCl solution. J. Chem. Phys. 2004;121:7840-7848.

[17] Laria D, Martí J, Guàrdia, E. Protons in supercritical water: a multistate empirical valence bond study. J. Am. Chem. Soc. 2004;126:2125-2134.

[18] Asthagiri D, Pratt LR, Kress, JD. Ab initio molecular dynamics and quasichemical study of $\mathrm{H}+$ (aq). Proc. Nat. Acad. Sci. USA. 2005;102:6704-6708.

[19] Botti A, Bruni F, Ricci MA, Soper AK. Eigen versus Zundel complexes in HCl-water mixtures. J. Chem. Phys. 2006;125:014508.

[20] Voth GA. Computer simulation of proton solvation and transport in aqueous and biomolecular systems. Acc. Chem. Res. 2006;39:143-150.

[21] Marx D. Proton transfer 200 years after von Grotthuss: Insights from ab initio simulations. ChemPhysChem. 2006; 7:1848-1870.

[22] Swanson JM, Maupin CM, Chen H, Petersen MK, Xu J, Wu Y, Voth GA. Proton solvation and transport in aqueous and biomolecular systems: insights from computer simulations. J. Phys. Chem. B. 2007;111:4300-4314.

[23] Lee SH, Rasaiah, JC. Proton transfer and the mobilities of the $\mathrm{H}+$ and $\mathrm{OH}$ ions from studies of a dissociating model for water. J. Chem. Phys. 2011;135:124505.

[24] Tahat A, Martí J. Dynamical aspects of intermolecular proton transfer in liquid water and low-density amorphous ices. Phys. Rev. E. 2014;89:052130.

[25] Wang C, Waje M, Wang X, Tang JM, Haddon RC, Yan, Y. Proton exchange membrane fuel cells with 


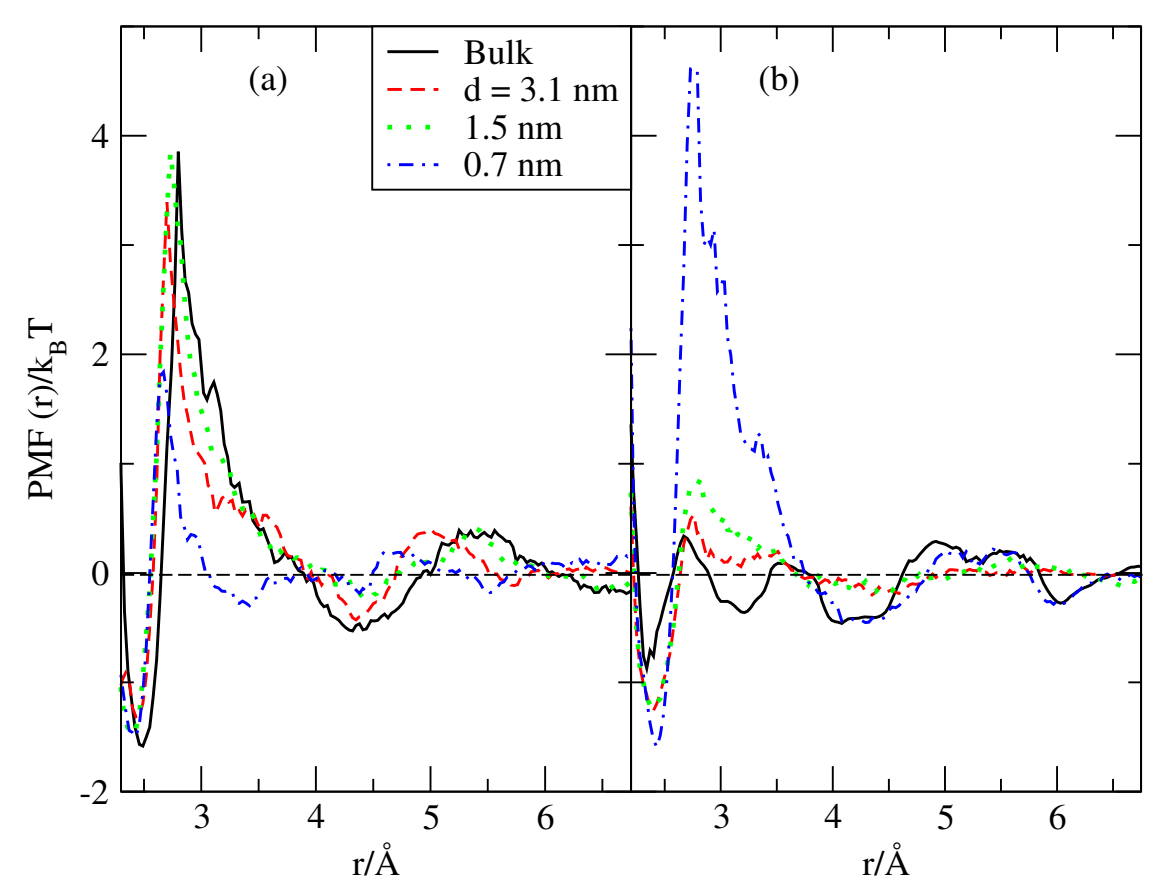

Figure 4. Potentials of mean force proton-water (in $k_{B} T$ ) at $200 \mathrm{~K}(\mathrm{a})$ and $600 \mathrm{~K}$ (b) for unconstrained water and for water inside graphene slabs of variable width.

carbon nanotube based electrodes. Nanolett. 2004;4:345-348.

[26] Spry DB, Goun A, Glusac K, Moilanen DE, Fayer MD. Proton transport and the water environment in nafion fuel cell membranes and aot reverse micelles. J. Am. Chem. Soc. 2007;129:8122-8130.

[27] Hynes JT, Klinman JP, Limbach H-H, Schowen RL (Eds.) Hydrogen-transfer reactions: John Wiley. New York; 2006.

[28] Chernyshev A, Cukierman S. Thermodynamic view of activation energies of proton transfer in various gramicidin A channels. Biophys. J. 2002;82:182-192.

[29] Le Caër S, Palmer DJ, Lima M, Renault JP, Vigneron G, Righini R, Pommeret S. Time-resolved studies of water dynamics and proton transfer at the alumina-air interface. J. Am. Chem. Soc. 2007;129:1172011729.

[30] Brewer ML, Schmitt UW, Voth GA. The formation and dynamics of proton wires in channel environments. Biophys. J. 2001;80:1691-702.

[31] Dellago C, Naor MM, Hummer G. Proton transport through water-filled carbon nanotubes. Phys. Rev. Lett. 2003;90:105902.

[32] Soper AK. Structural transformations in amorphous ice and supercooled water and their relevance to the phase diagram of water. Mol. Phys. 2008;106:2053-2076.

[33] Vega C, Abascal JL, Conde MM, Aragones JL. What ice can teach us about water interactions: a critical comparison of the performance of different water models. Faraday Discuss. 2009;141: 251-276.

[34] Cao Z, Peng Y, Yan T, Li S, Li A, Voth GA. Mechanism of fast proton transport along one-dimensional water chains confined in carbon nanotubes. J. Am. Chem. Soc. 2010;132:11395-11397.

[35] Hummer G, Rasaiah JC, Noworyta JP. Water conduction through the hydrophobic channel of a carbon nanotube. Nature. 2001;414:188-190. 
[36] Wolf MG, Groenhof G. Explicit proton transfer in classical molecular dynamics simulations. J. Comput. Chem. 2014;35:657-671.

[37] Bankura A, Chandra A. Hydroxide ion can move faster than an excess proton through one-dimensional water chains in hydrophobic narrow pores. J. Phys. Chem. B. 2012;116:9744-9757.

[38] Clark II JK, Paddison SJ. Ab initio molecular dynamics simulations of water and an excess proton in water confined in carbon nanotubes. Phys. Chem. Chem. Phys. 2014;16:17756-17769.

[39] Mahadevan TS, Garofalini, SH. Dissociative water potential for molecular dynamics simulations. J. Phys. Chem. B. 2007;111:8919-8927.

[40] Wiedemair MJ, Hitzenberger M, Hofer, TS. Tuning the reactivity of a dissociative force field: proton transfer properties of aqueous $\mathrm{H} 3 \mathrm{O}+$ and their dependence on the three-body interaction. Phys. Chem. Chem. Phys. 2015; 17:10934-10943.

[41] Bankura A, Chandra A. Proton transfer through hydrogen bonds in two-dimensional water layers: A theoretical study based on ab initio and quantum-classical simulations. J. Chem. Phys. 2015;142:044701.

[42] Lockwood GK, Garofalini, SH. Lifetimes of excess protons in water using a dissociative water potential. J. Phys. Chem. B. 2013;117:4089-4097.

[43] Tahat A, Martí J. Proton transfer in liquid water confined inside graphene slabs. Phys. Rev. E. 2015;92:032402.

[44] Coulson CA, Danielsson U. Ionic and covalent contributions to the hydrogen bond. Part I. Ark. Fys. 1954;8:239-244.

[45] Mulliken RS. Charge transfer complexes. J. Chim. Phys. 1964;61:20.

[46] Aqvist J, Warshel A. Simulation of enzyme reactions using valence bond force fields and other hybrid quantum/classical approaches. Chem. Rev. 1993;93:2523-2544.

[47] Warshel A. Computer Modeling of Chemical Reactions in Enzymes and Solutions: John Wiley. New York; 1980.

[48] Vuilleumier R, Borgis D. Quantum dynamics of an excess proton in water using an extended empirical valence-bond Hamiltonian. J. Phys. Chem. B. 1998;102:4261-4264.

[49] Vuilleumier R, Borgis D. An extended empirical valence bond model for describing proton transfer in $\mathrm{H}+(\mathrm{H} 2 \mathrm{O}) \mathrm{n}$ clusters and liquid water. Chem. Phys. Lett. 1998;284:71-77.

[50] Vuilleumier R, Borgis D. Transport and spectroscopy of the hydrated proton: a molecular dynamics study. J. Chem. Phys. 1999;111: 4251-4266.

[51] Lobaugh J, Voth GA. The quantum dynamics of an excess proton in water. J. Chem. Phys. 1996;104:2056-2069.

[52] Schmitt UW, Voth GA. Multistate empirical valence bond model for proton transport in water. J. Phys. Chem. B. 1998;102:5547-5551.

[53] Schmitt UW, Voth GA. The computer simulation of proton transport in water. J. Chem. Phys. 1999;111:9361-9381.

[54] Cuma M, Schmitt UW, Voth GA. A multi-state empirical valence bond model for acidbase chemistry in aqueous solution. Chemi. Phys. 2000;258:187-199.

[55] Day TJ, Soudackov AV, Cuma M, Schmitt UW, Voth GA. A second generation multistate empirical valence bond model for proton transport in aqueous systems. J. Chem. Phys. 2002;117:5839-5849.

[56] Sagnella DE, Tuckerman ME. An empirical valence bond model for proton transfer in water. J. Chem. Phys. 1998;108:2073-2083.

[57] Walbran S, Kornyshev AA. Proton transport in polarizable water. J. Chem. Phys. 2001;114:1003910048.

[58] Biswas R, Tse YL, Tokmakoff A, Voth GA. Role of Presolvation and Anharmonicity in Aqueous Phase Hydrated Proton Solvation and Transport. J. Phys. Chem. B. 2015;120:1793-1804.

[59] Dang LX, Pettitt BM. Simple intramolecular model potentials for water. J. Phys. Chem. 1987;91:33493354.

[60] Gordillo MC, Martí J. Hydrogen bond structure of liquid water confined in nanotubes. Chem. Phys. Lett. 2000;329: 341-345.

[61] Spohr E. Effect of electrostatic boundary conditions and system size on the interfacial properties of water and aqueous solutions. J. Chem. Phys. 1997;107:6342-6348.

[62] Frenkel D, Smit B. Understanding Molecular Simulation: Academic Press. San Diego; 2002.

[63] Hub JS, de Groot BL, Grubmüller H, Groenhof G. Quantifying artifacts in Ewald simulations of inhomogeneous systems with a net charge. J. Chem. Theor. Comput. 2014;10:381-390. 
[64] Zundel G, Metzger H. Energiebänder der tunnelnden überschuss-protonen in flüssigen säuren. Eine IRspektroskopische untersuchung der natur der gruppierungen H5O2+. Zeits. Phys. Chemie. 1968;58:225245.

[65] Eigen M, De Maeyer L. Self-dissociation and protonic charge transport in water and ice. Proc. R. Soc. London. 1958;A247:505-533.

[66] Tuckerman M, Laasonen K, Sprik M, Parrinello M. Ab initio molecular dynamics simulation of the solvation and transport of hydronium and hydroxyl ions in water. J. Chem. Phys. 1995;103:150-161.

[67] Tuckerman M, Laasonen K, Sprik M, Parrinello M. Ab initio molecular dynamics simulation of the solvation and transport of $\mathrm{H} 3 \mathrm{O}+$ and OH-ions in water. J. Phys. Chem. 1995;99:5749-5752.

[68] Tahat A, Martí J, Khwaldeh A, Tahat K. Pattern recognition and data mining software based on artificial neural networks applied to proton transfer in aqueous environments. Chin. Phys. B 2014;23:046101.

[69] Moon E-S, Yoon J, Kang H. Energy barrier of proton transfer at ice surfaces. J. Chem. Phys. 2010;133:044709.

[70] Park SC, Moon ES, Kang H. Some fundamental properties and reactions of ice surfaces at low temperatures. Phys. Chem. Chem. Phys. 2010;12:12000-12011.

[71] Kim JH, Kim Y-K, Kang H. Proton transfer and H/D isotopic exchange of water molecules mediated by hydroxide ions on ice film surfaces. J. Chem. Phys. 2009;131:044705.

[72] Luz Z, Meiboom S. The activation energies of proton transfer reactions in water. J. Am. Chem. Soc. 1964;86:4768-4769. 